\title{
Alternative Imaging Modalities to DSA in the Acute Phase of Unruptured Vertebral Artery Dissection
}

\author{
Vasileios Arzoglou, a, Stamatia Potsi ${ }^{\mathrm{b}}$, Matthaios Savvidis ${ }^{\mathrm{c}}$, Panagiotis Selviaridis ${ }^{\mathrm{a}}$, Athanasios \\ Spiliotopoulos ${ }^{\mathrm{a}}$
}

\begin{abstract}
Minor neck movements are a known cause of vertebral artery dissection even without any evidence of trauma. However, there are limited reports of unilateral vertebral artery dissection in a trauma patient evolving to a fatal posterior fossa infarction within one day, in the absence of any neurological deficit or a focal lesion on brain CT. We report such a case and comment on the overall efficiency of diagnostic alternatives to classic angiography that can be used in the acute phase of unruptured vertebral artery dissection. We present a case of a healthy 55 year old trauma patient with initial good neurological presentation and normal non-infused brain $\mathrm{CT}$ who deteriorated 16 hours following injury and deceased the following day due to a massive posterior fossa infarction and extended cerebral edema caused by vertebral artery dissection. We conclude that any trauma patient with a persistent, even mild occipital headache or neck pain can be a candidate for vertebral artery dissection. Such pathology requires both a high index of suspicion and the proper imaging modality. Vertebral artery dissection can be efficiently visualized by multisection CT angiography which is fast, accurate and non-invasive. This modality can be an alternative to classic angiography especially in emergency settings where it may not be easily available.
\end{abstract}

Keywords: Vertebral artery dissection; Infarction; Head injury; Imaging

Manuscript accepted for publication May 10, 2012

${ }^{a} 1$ st Neurosurgical Department - Aristotle's University Hospital of Thessaloniki AHEPA, Greece

${ }^{\mathrm{b}}$ Department of Radiology - Interbalkan Medical Center, Thessaloniki, Greece

'Orthopaedic Department - 424 Military Hospital of Thessaloniki,

Greece

${ }^{\mathrm{d}}$ Corresponding author: Vasileios Arzoglou, Neurosurgical

Department - 6th floor - AHEPA University Hospital, Stilponos

Kiriakidi 1, Thessaloniki, PC 54636, Greece.

Email: arzoglouvasilis@yahoo.com

doi:10.4021/jmc696w

\section{Introduction}

Vertebral artery dissection (VAD) results when a layer of the vertebral artery wall (intimae or media) is torn, or when there is direct hemorrhage from the vasa vasorum. Subsequent hematoma can dissect through the media under the intimae or the adventitia, resulting either in lumen narrowing or in a pseudoaneurysm formation. Clinical manifestations begin when disrupted blood flow followed by luminal thrombosis and distal embolism are established, or when there is rupture of the arterial wall. VAD can occur spontaneously, after trauma or even after minor stress such as sneezing or coughing [1]. It is a well known factor of posterior fossa infarction in adults with increasing incidence in the last years [2]. This increase is not only attributed to the absolute increase of incidence, but also to the growing familiarity with this pathology and the improvement in diagnostic imaging techniques. Almost $61 \%$ of VAD are referred to be spontaneous, $30 \%$ take place after trauma and $9 \%$ are a result of cervical manipulation at physiotherapy $[1,3]$. The incidence of VAD after blunt trauma ranges from $0.20 \%$ to $0.77 \%$ [4] carrying a mortality rate of $10 \%$ [3] in the acute phase. The annual incidence of spontaneous vertebral artery dissection is 1 to 1.5 per 100,000 population [5], and female to male ratio varies between $3 / 1$ to $5 / 1[1,3]$. When reviewing the literature that percentages can vary a lot. Apart from that, after several reports of VAD following minimal neck stress such as sneezing, coughing or ceiling painting, it is obvious that the term spontaneous dissection can be questionable sometimes.

In relation to the age and contrary to the incidence of pure atherothrombotic lesions of vertebro-basilar circulation, VAD is responsible for $20 \%$ of ischemic infarcts in adults younger than 45 years of age [2]. Recent reports advocate cervical mechanical stress in sports or in ordinary everyday activities as an increasingly recognized cause of dissections. Therefore new syndromes of vertebral artery dissection have appeared in the literature such as the ceiling painting stroke, beauty parlour stroke, bottoms up stroke or the golf player's stroke [1, 6-9]. On the other hand references of spontaneous dissections in patients with cystic medial necrosis, Bechget's disease, rheumatoid arthritis, giant-cell arteritis, fibromus- 


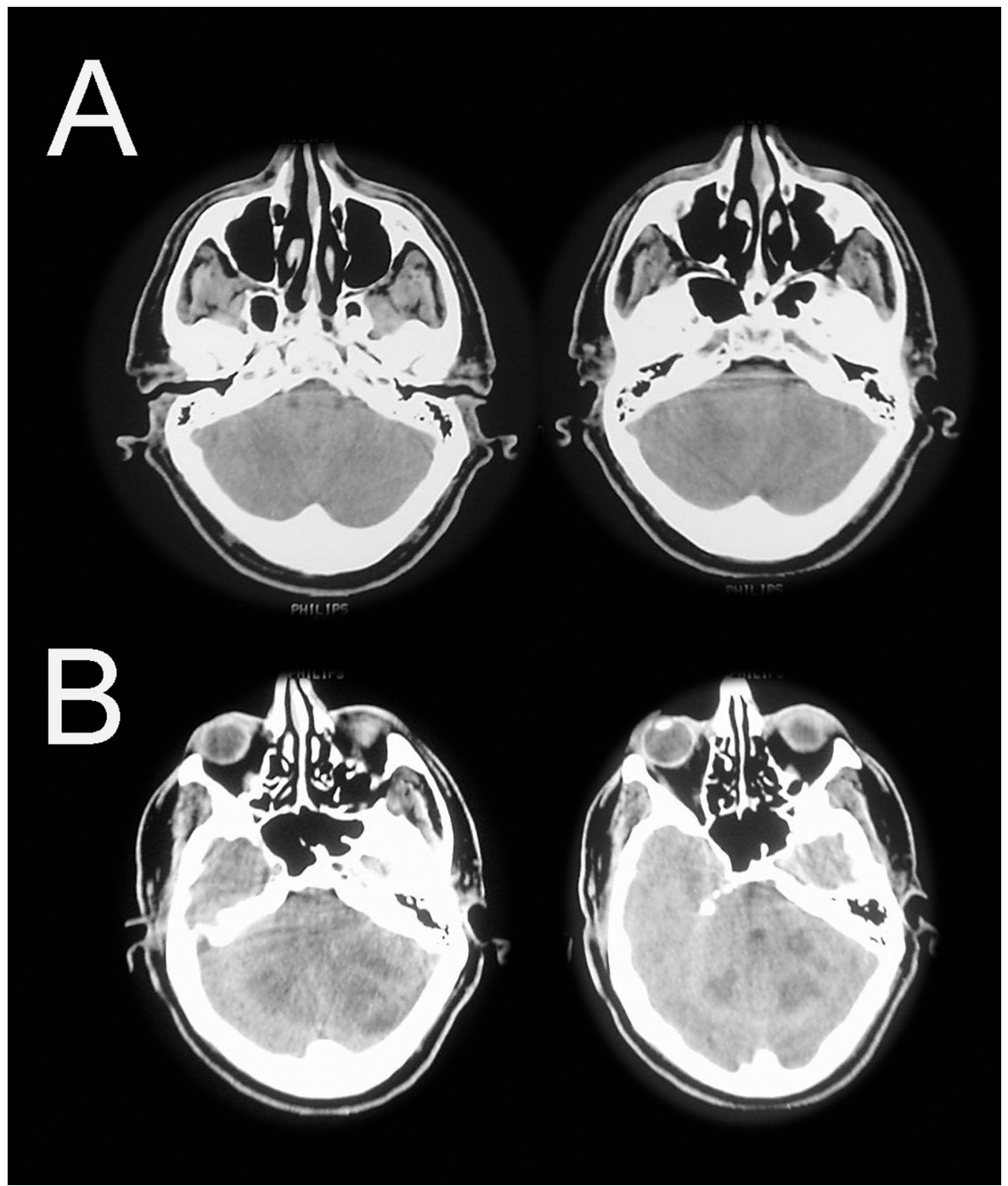

Figure 1. A. CT at admission; B: Control CT 16 hours post trauma showing a massive infarction and extended cerebral edema.

cular dysplasia , intracranial aneurysms, $\alpha 1$-antitrypsin deficiency, Ehlers-Danlos type-IV syndrome, Marfan syndrome, autosomal dominant polycystic kidney disease and osteogenesis imperfecta type-I [1,3,9-11] may reveal some of the predisposing factors for VAD.

\section{Case Report}

A 55 years old male patient without any previous medical history was admitted to a local hospital after a road traffic accident. The patient was seen by witnesses to have walked out of his car without having lost his consciousness. In the emergency room he was complaining of occipital headache, without any signs of trauma or neurologic deficit. Non-infused CT of the brain (Fig. 1) didn't reveal any pathology, but the patient was admitted for observation due to persisting occipital headache. After 16 hours he started deteriorating and within 1 hour he ended up in coma and bilateral pupillary dilatation. Control CT scan revealed a posterior fossa infarction and extended cerebral edema (Fig. 1). The patient was transferred to a tertiary medical center where a digital substraction angiography (DSA) of the vertebral and carotid arteries was performed. DSA revealed a dissection at the $3 \mathrm{rd}$ level of the vertebral artery, several thrombi and lack of cerebral circulation (Fig. 2). No neurosurgical intervention was performed and the patient deceased 24 hours later.

\section{Discussion}

Clinical presentation of acute VAD may not be different from that of mild head injuries or muscular cervical strain. Such pathologies may co-exist and symptoms usually over- 


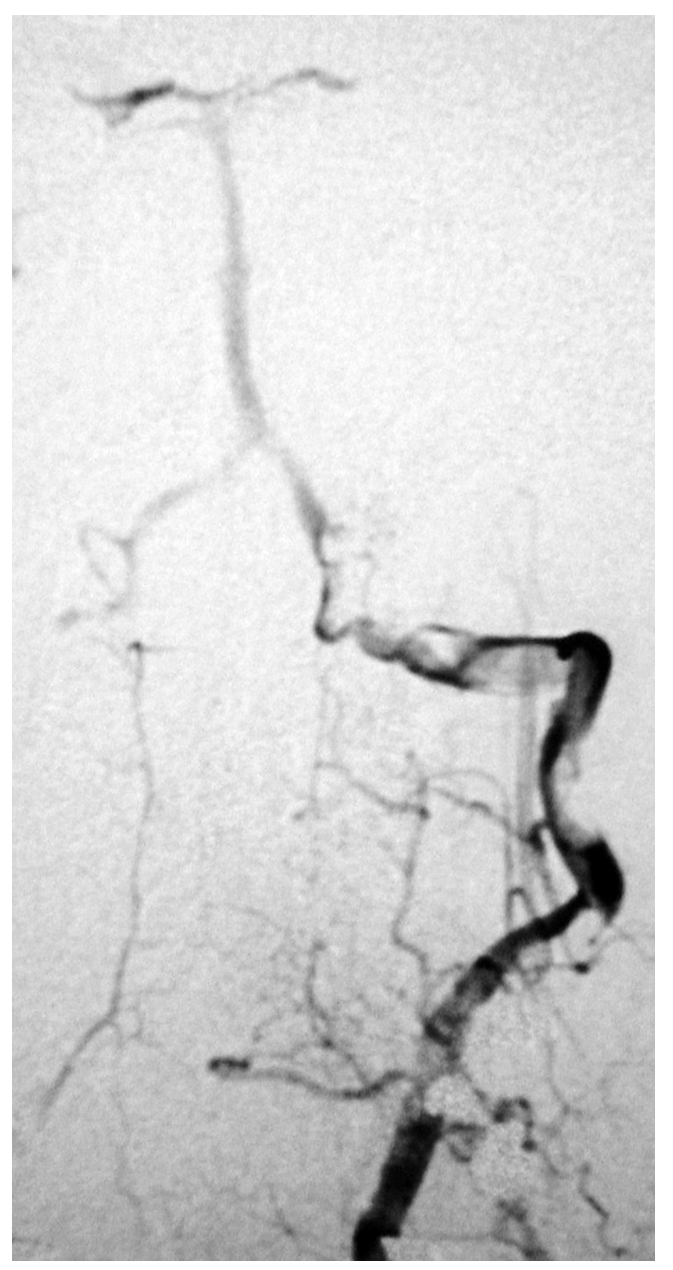

Figure 2. DSA of the patient. Dissection of vertebral artery with presence of thrombi and distal occlusion.

lap making the diagnosis difficult. Even until today, the golden standard for the diagnosis and follow-up of VAD that all other imaging modalities are compared to is DSA. Unfortunately it is an invasive technique and it cannot be easily accessed in all health care settings. On the other hand, noninfused $\mathrm{CT}$ of the brain, which is the first line examination for traumatic head injuries, is only able to diagnose complications of VAD like subarachnoid haemorrhage and infarction. Intraluminal lesions that take place in the acute phase of an unruptured VAD can be easier distinguished after contrast medium administration.

Multisection CT angiography is a minimally invasive technique that can visualize both the arterial lumen and its wall. When maximum intensity projection or curved planar reconstructions are used, it can further provide images equivalent to DSA in means of both resolution and contrast [12]. Multisection CTA compared to DSA can be of high accuracy reaching values of $95 \%$ in positive prognostic value and $100 \%$ in negative prognostic value [12]. MRI-MRA combined with CTA can provide better diagnostic results than DSA because specific radiographic signs such as pseudoaneurysm, intimal flap, or a double-lumen appearance that are diagnostic for VAD with DSA, are usually seen in less than $10 \%$ of cases [13]. Apart from that, while being diagnostic for DSA they are also coexisting findings in $10 \%$ of atherosclerotic vessels or recanalisating vessels previously partially occluded by emboli [12]. CT and MRA have also the ability to detect the intraluminal haematoma per se which can be very helpful when subtle luminal abnormalities follow dissection [12]. Multisection CTA alone may reach values of $100 \%$ in sensitivity and $98 \%$ in specificity in the diagnosis of VAD $[12,15]$. In delineating a very small lumen with minimal flow, multisection CT angiography has been reported to be inferior to conventional angiography. A stenotic dissection has been misinterpreted as a tapered occlusion in a report, because of poor visualization of the threadlike remaining lumen on CT images [12]. The reason proposed by the reporting author was the different injection pressures between conventional angiography and CT angiography (direct arterial versus venous injection).

When MRI is used alone for the diagnosis of VAD, several false positive results have been reported from artifacts. The undifferentiating intraluminal signal of VAD in the acute phase and the accuracy of small structures such as the vertebral artery combined with artifacts from the venous and adipose tissue signals surrounding the vertebral artery, have raised concerns about MRI's accuracy in detecting VAD [15, 16]. Venous plexus may have a semilunar appearance, and slow flow in its lumen may give rise to high signal intensity on both MRI and MRA, creating an image mimicking a dissection $[15,17]$. It has been suggested that saturation slabs in conjunction with MRA completely suppress flow related high signal, thus distinguishing it from high signal due to an intramural haematoma which cannot be suppressed by saturation slabs $[17,18]$.

The second tissue that may falsely present as a dissection is fat that directly surrounds vertebral arteries. This fat also gives rise to high signal intensity, but using fat suppression technique, it can be adequately differentiated from intramural haematoma [15]. Furthermore, the usual diameter asymmetry of vertebral arteries, turbulence and magnetic susceptibility near sharp vessel turns can also cause false positive MRA results $[8,15]$. In some patients, MRI couldn't distinguish between intraluminal thrombus and intramural hematoma, leading to false conclusions. Additionally mural thrombus within aneurysms or occluded vessels may resemble an intramural hematoma on MR images [16]. In this sense, MR imaging may not be a highly specific examination [19]. The sensitivity and specificity for diagnosing VAD with MR imaging can be $20 \%$ and $100 \%$ respectively while for MRA they can be $60 \%$ and $98 \%$ [20]. MRI and MRA tend to have a better diagnostic ability in subacute or early chronic intramural hematoma because of its conspicuous high signal intensity on T1-weighted images. More sophisticated tech- 
niques such as the time of flight (TOF) MRA may finally become a safe non-invasive alternative to conventional angiography in long term follow up of patients that suffered VAD. Nevertheless, neither MRI-MRA nor CT is able to completely differentiate an intramural hematoma in an occluded dissection from a mural thrombus in an occluded vessel [12].

It is hard to conclude to one technique, that it's the best and that can safely replace all the others. Each one has its benefits and pitfalls, and they are all differently available around primary health care settings. Although they can be complementary to each other it is impossible to expose each patient to all these tests. Therefore the optimal choice of the diagnostic modality for VAD must be carefully made depending on the time of the suspected dissection, the imaging modalities available and the familiarity with this pathology.

\section{Conclusions}

In trauma patients with a persistent, even mild headache, VAD can not be excluded from the differential diagnosis. Diagnosis of VAD in the acute phase requires a high degree of suspicion, and a proper imaging modality to document it. Multisection CT can have a higher specificity in the acute phase of VAD due to high accuracy and absence of artifacts related to intraluminal flow, while on the other hand MRI and MRA tend to have a better diagnostic ability in depicting a subacute or early chronic intramural haematoma. Multisection CT angiography is a safe and accurate technique that should be performed in the acute phase of all suspected VAD patients. It is considered an efficient alternative to DSA providing a non-invasive modality that can be easily accessed in most emergency settings.

\section{Abbreviations}

DSA: Digital Substraction Angiography; CT: Computerized Tomography; CTA: Computerized Tomography Angiography; VAD: Vertebral Artery Dissection; MRA: Magnetic Resonance Angiography; MR: Magnetic Resonance; TOF: Time Of Flight.

\section{References}

1. Haneline MT, Lewkovich GN. An analysis of the etiology of cervical artery dissections: 1994 to 2003. J Manipulative Physiol Ther. 2005;28(8):617-622.

2. Bogousslavsky J, Regli F. Ischemic stroke in adults younger than 30 years of age. Cause and prognosis. Arch Neurol. 1987;44(5):479-482.

3. Lang E, Afilalo M. Dissection, vertebral artery. http:// www.emedicine.com. Accessed November 1, 2009.
4. Inamasu J, Guiot BH. Vertebral artery injury after blunt cervical trauma: an update. Surg Neurol. 2006;65(3):238245; discussion 245-236.

5. Redekop GJ. Extracranial carotid and vertebral artery dissection: a review. Can J Neurol Sci. 2008;35(2):146-152.

6. Weintraub MI. Beauty parlor stroke syndrome: report of five cases. JAMA. 1993;269(16):2085-2086.

7. HartRG, Easton JD. Dissections. Stroke. 1985;16(6):925927.

8. Hart RG. Vertebral artery dissection. Neurology. 1988;38(6):987-989.

9. Rubinstein SM, Peerdeman SM, van Tulder MW, Riphagen I, Haldeman S. A systematic review of the risk factors for cervical artery dissection. Stroke. 2005;36(7):15751580 .

10. Schievink WI, Bjornsson J, Piepgras DG. Coexistence of fibromuscular dysplasia and cystic medial necrosis in a patient with Marfan's syndrome and bilateral carotid artery dissections. Stroke. 1994;25(12):2492-2496.

11. Schievink WI, Bjornsson J, Parisi JE, Prakash UB. Arterial fibromuscular dysplasia associated with severe alpha 1-antitrypsin deficiency. Mayo Clin Proc. 1994;69(11):1040-1043.

12. Chen CJ, Tseng YC, Lee TH, Hsu HL, See LC. Multisection CT angiography compared with catheter angiography in diagnosing vertebral artery dissection. AJNR Am J Neuroradiol. 2004;25(5):769-774.

13. Provenzale JM. Dissection of the internal carotid and vertebral arteries: imaging features. AJR Am J Roentgenol. 1995;165(5):1099-1104.

14. Leclerc X, Godefroy O, Salhi A, Lucas C, Leys D, Pruvo JP. Helical CT for the diagnosis of extracranial internal carotid artery dissection. Stroke. 1996;27(3):461-466.

15. Bloem BR, Van Buchem GJ. Magnetic resonance imaging and vertebral artery dissection. J Neurol Neurosurg Psychiatry. 1999;67(5):691-692.

16. Kitanaka C, Tanaka J, Kuwahara M, Teraoka A. Magnetic resonance imaging study of intracranial vertebrobasilar artery dissections. Stroke. 1994;25(3):571-575.

17. Miaux Y, Cognard C, Martin-Duverneuil N, Weill A, Savin D, Chiras J. Flow-related enhancement in the vertebral plexus mimicking an intramural hematoma. AJNR Am J Neuroradiol. 1996;17(1):191-192.

18. Dumas JL, Stanescu R, Goldlust D, Brugieres P. Vertebral vein imaging with MR angiography. AJNR Am J Neuroradiol. 1997;18(6):1190-1192.

19. McCormick GF, Halbach VV. Recurrent ischemic events in two patients with painless vertebral artery dissection. Stroke. 1993;24(4):598-602.

20. Levy C, Laissy JP, Raveau V, Amarenco P, Servois V, Bousser MG, Tubiana JM. Carotid and vertebral artery dissections: three-dimensional time-of-flight MR angiography and MR imaging versus conventional angiography. Radiology. 1994;190(1):97-103. 